\section{Funktions- und organerhaltende Therapie beim Blasenkarzinom}

$D_{\mathrm{i},}^{\mathrm{i}}$ ie kombinierte Radiochemotherapie ist laut Prof. Frederik Wenz, Mannheim, ein wichtiger Baustein der multimodalen, organ- und funktionserhaltenden Therapie des muskelinvasiven Harnblasenkarzinoms. Dabei sei die Radiochemotherapie nicht als Alternative zur interventionellen urologischen Versorgung eines Harnblasenkarzinoms, sondern als adjuvante oder postoperative Therapie nach transurethraler Resektion (TUR) als Alternative zur Zystektomie zu sehen. Nachdem der Tumor im Rahmen einer TUR möglichst komplett (R0) reseziert wurde, sollte nach 2-6 Wochen eine Radio- oder Radiochemotherapie mit ca. 50-
60 Gy in konventioneller Fraktionierung erfolgen. Bei kurativer Intention sei eine simultane Chemotherapie grundsätzlich indiziert. Eine erste randomisierte Studie (Radiotherapie mit und ohne Cisplatin) habe für die kombinierte Radiochemotherapie einen signifikanten Vorteil hinsichtlich lokaler Kontrolle sowie einen Trend zu besseren Überlebensraten gezeigt.

Nach dem Erlanger-Protokoll erhalten Patienten 5-mal $25 \mathrm{mg} / \mathrm{m}^{2} \mathrm{KO}$ Cisplatin an Tag 1-5 der ersten und der fünften Radiotherapiewoche, erklärte Wenz. Bei einer Behandlung nach dem Boston/ RTOG-Schema erhalten Patienten 70 $\mathrm{mg} / \mathrm{m}^{2} \mathrm{KO}$ Cisplatin alle drei Wochen (d.h. 3-mal während der Radiotherapie). Dieses Regime sei ähnlich effektiv, berge aber ein höheres Komplikationsrisiko, so Wenz. Die radiosensibilisierende Chemotherapie werde vorrangig unter dem Aspekt der verbesserten lokalen Kontrolle eingesetzt. Die Fernmetastasierung sei eher von untergeordneter Bedeutung.

Akute strahlenbedingte Nebenwirkungen betreffen vor allem die Harnblase und den benachbarten Enddarm. Eine akute radiogene Zystitis tritt meist nach der Hälfte der Bestrahlung auf. Bei den angewandten Dosen und den modernen Bestrahlungstechniken seien chronische Spätfolgen selten. Im Erlanger Kollektiv wurden relevante Spätfolgen (Grad 3-4) bei $5 \%$ beobachtet. Laut Wenz hatten etwa $75-80 \%$ der Langzeitüberlebenden eine normale Blasenfunktion. Doris Berger

\section{Erweiterte Therapieoptionen beim benignen Prostatasyndrom}

$\mathrm{M}$ edikamentöse Kombinationen verschiedener Wirkmechanismen sind in der Behandlung von Patienten mit benignem Prostatasyndrom (BPS) effektiver als die Einzelsubstanzen. Eine neue und noch nicht in den Leitlinien enthaltene Option ist die Kombination eines PDE5-Inhibitors mit einem 5-alpha-Reduktasehemmer. Damit habe sich in einem Direktvergleich der Kombination Tadalafil/ Finasterid mit Finasterid/Placebo eine signifikante Verbesserung beim Symptomenscore IPSS zugunsten der Kombination ergeben, erklärte Prof. Rolf Muschter, Rotenburg, und zwar unabhängig vom Vorliegen einer erektilen Dysfunktion (ED). Lag eine ED zusätzlich vor, profitierten die Männer zudem von einer deutlich verbesserten erektilen Funktion.

Zwar würden derzeit weitere medikamentöse Therapien erprobt, jedoch lägen bislang für keine der neuen Substanzen ausreichend Daten vor, erklärte Muschter. Therapeutische Neuigkeiten auf dem Gebiet der BPS sind daher vor allem instrumenteller Art, was Destruktions-, Ablations- und Retraktionstechniken angeht, aber auch die von interventionellen Radiologen praktizierte Embolisation von Prostataarterien. Eine Ablations- technik ist die sonografiegestützte und roboterassistierte Water-Jet-Hydrodissektion. Mit einem Hochgeschwindigkeits-Wasserstrahl wird Prostatadrüsengewebe entfernt, wobei Kapsel und Gefäße geschont werden. Hitze entsteht bei dem Verfahren nicht. Das adenomatöse Zielgewebe wird nach Angaben des Herstellers entsprechend der präoperativen Planung exakt entfernt, das Ganze dauert nur wenige Minuten. Beim EAU wurden bereits erste Ergebnisse einer Pilotstudie vorgestellt. Wasser wird aber auch in Form von heißem Dampf zur Ablation eingesetzt. Mit einem zystoskopartigen Gerät wird der Wasserdampf transurethral und durch eine Nadel für wenige Sekunden ins Zielgewebe geleitet. So wird eine Kolliquationsnekrose bewirkt.

Auch Geweberetraktoren sind eine Option. Dabei werden minimalinvasiv mehrere permanente Implantate eingesetzt, die wie Raffhalter an Vorhängen die Lappen der vergrößerten Prostata offen halten, um die Blockierung für den Urinfluss aufzuheben. Prostatagewebe wird weder entfernt noch destruiert. Vorteil ist der Erhalt der Sexualfunktion und ein für den Patienten rasch spürbares Ergebnis, wenngleich nach aktuellen Daten die transurethrale Resektion einen vergleichsweise besseren maximalen Harnfluss erzielt. In Bezug auf Lebensqualität und IPSS sind die Verfahren vergleichbar.

Die selektive Embolisation von Prostataarterien ist ein Verfahren, das von interventionellen Radiologen eingesetzt wird. Der Zugang erfolgt über die Leistenarterie in Lokalanästhesie. Unter fluoroskopischer Kontrolle wird ein Mikrokatheter (0,7 mm Durchmesser) bis in das prostataversorgende Gefäß geführt, dort werden dann Microspheren einer Größe von $200-400 \mu \mathrm{m}$ platziert. „Meistens sind drei Arterien pro Seite zu embolisieren“, berichtete Muschter. Daraufhin beginnt die Prostata zu schrumpfen. In der Literatur werden bereits gute kurz- bis mittelfristige Ergebnisse in Bezug auf die typische BPS-Symptomatik berichtet.

Vorteile dieser instrumentellen Verfahren: Sie können oft ambulant vorgenommen werden und zum Teil sehr rasch $\mathrm{zu}$ Verbesserungen führen. Muschter wollte aufgrund noch nicht ausreichender Daten die einzelnen Verfahren aber nicht bewerten. „Ich bin überzeugt, dass das Ende der Fahnenstange noch lange nicht erreicht ist“, so Muschter. Weitere Ideen werden das Feld der BPS-Therapie auch künftig bereichern. Eine andere Frage ist, inwieweit gesetzlich versicherte Patienten solche Verfahren erstattet bekommen werden.

Thomas Meissner 